\title{
Un outil peu connu mais efficient au service du principe ALARA : ISOE (Information System on Occupational Exposure)
}

\author{
M. CROQ*, S. MUNDIGL**, L. D'ASCENZO***, T. LAZO**, C. LEFAURE***
}

(Manuscrit reçu le 30 octobre 2000, accepté le lo février 2001)

RÉSUMÉ Le système d'information sur les expositions professionnelles aux rayonnements ionisants ou ISOE (Information System on Occupational Exposure) est un programme qui a vu officiellement le jour Ie $1^{\text {er }}$ janvier 1992 sous l'impulsion de l'agence de l'énergie nucléaire (AEN). Ce programme a pour but de répondre au besoin des centrales nucléaires de production d'électricité et des autorités de sûreté de disposer d'un outil de comparaison et d'information sur les pratiques en matière d'optimisation (principe ALARA) dans les différents pays membres. Actuellement, plus de $90 \%$ des centrales nucléaires commerciales de part le monde sont affiliées à ce programme. La structure fonctionnelle du programme ISOE s'articule autour d'un comité directeur et de quatre centres techniques (Europe, Amérique du nord, Asie et AIEA). Le programme ISOE est constitué de bases de données concernant I'exposition des travailleurs et d'un réseau de communication et d'échange. Les bases de données permettent de réaliser des analyses et des comparaisons en matière de pratique, soit sur un plan purement factuel, soit au contraire de manière analytique. Ceci permet de profiter au mieux de l'expérience commune et donc de se rapprocher le plus possible du « achievable » du principe ALARA.

ABSTRACT An efficient but little known tool for the application of the ALARA principle: ISOE (Information System on Occupational Exposure).

The Information System on Occupational Exposure to ionizing radiation (ISOE) is a program launched by the Nuclear Energy Agency (NEA) in 1992. It was developed to address the needs of nuclear power plants and safety authorities as a comparison and information tool on practices in terms of optimization (ALARA principle), in NEA member countries. To date, more than $90 \%$ of the operating nuclear power plants in the world are participating in this program, which is organized in a decentralized form, with a steering group and four technical centers (Europe, Asia, north America and IAEA). The ISOE program includes an occupational exposure database and an information exchange and communication network. The databases permit the preparation of studies and comparisons of practices, either in a factual or analytical way. Thus, the ISOE program favors exchanges to the benefit of all participants and helps to bring operational radiation protection ever closer towards the "achievable" of ALARA.

\footnotetext{
* Service de protection radiologique des armées, I his rue du lieutenant Raoul Batany, 92 I40 Clamart, France.

** Agence de l'OCDE de l'énergie nucléaire, 12 boulevurd dé îles. 92130 Issy-les-Moulineaux, France.

*** Centre d'étude sur l'évaluation de la protection dans le domaine nucléaire, route du panorama, BP 48, 92263 Fontenay allx-Roses Cedex, France.
} 


\section{Introduction}

Le système d'information sur les expositions professionnelles aux rayonnements ionisants (Information System on Occupational Exposure, ISOE) a vu le jour officiellement le $1^{\text {cr }}$ janvier 1992, après une phase d'essai de deux ans, sous l'impulsion de l'Agence de l'énergie nucléaire (AEN) de l'Organisation de coopération et de développement économiques (OCDE). Le développement et la mise en place de ce programme avaient pour but de répondre au besoin des centrales nucléaires de production d'électricité et des autorités de sûreté de disposer d'un outil de comparaison et d'information sur les pratiques en matière d'optimisation (principe ALARA) dans les différents pays membres de l'AEN. Dès 1993, l'Agence internationale de l'énergie atomique (AIEA), devant l'intérêt évident d'un tel programme, s'est associée au développement d'ISOE permettant ainsi aux pays non-membres de l'AEN de pouvoir y participer.

\section{Organisation et structure du programme}

\subsection{Organisation fonctionnelle du programme international ISOE}

Le programme ISOE est dirigé par un comité directeur qui est responsable de l'ensemble du programme et de sa stratégie générale. Ce comité ne se réunit qu'une fois l'an, dans l'intervalle il est représenté par un bureau exécutif qui s'appuie sur le secrétariat conjoint AEN/AIEA pour faire fonctionner le programme. Le secrétariat conjoint gère et organise le transfert d'informations entre le comité directeur, les groupes de travail et les différents centres techniques (Fig. 1) ; il est par ailleurs chargé de la rédaction des rapports annuels qui fournissent les principaux résultats du programme ISOE et de documents d'information (voir références).

Les centres techniques sont au nombre de quatre, Asie, Europe, Amérique du nord et AIEA (pays non-membres de l'AEN); ils sont chargés de récupérer les données concernant les réacteurs participants au programme dans leur zone et de réaliser des lettres d'information concernant les pratiques et leurs évolutions. Le centre d'étude sur l'évaluation de la protection dans le domaine nucléaire (CEPN) situé à Fontenay-aux-Roses héberge le centre technique européen ; celui-ci est par ailleurs chargé du développement, de la mise à jour et de la distribution des différentes bases de données. Les centres techniques européens et américains organisent en alternance tous les deux ans un séminaire international sur la gestion des expositions professionnelles. 


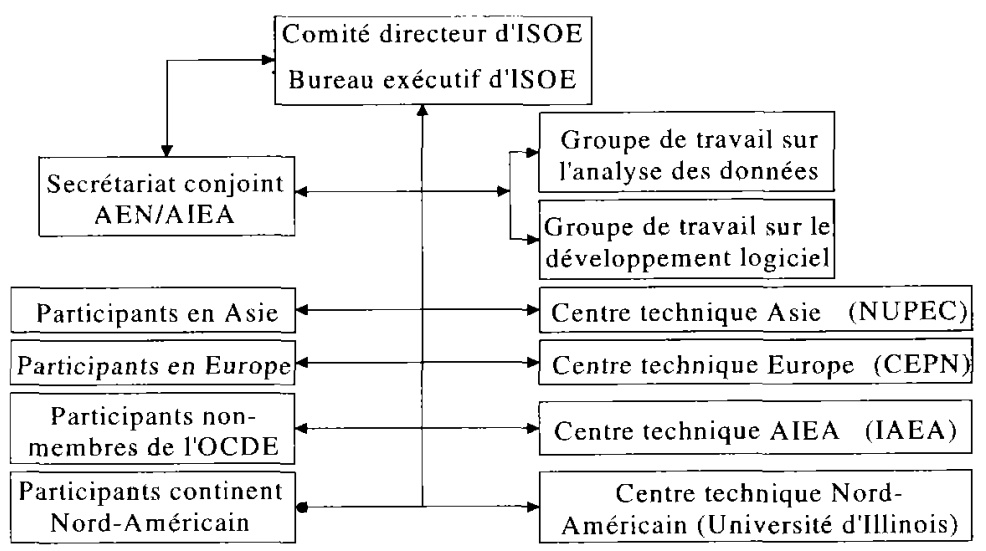

Figure 1 - Organisation générale du programme ISOE.

General organization of the ISOE program.

\subsection{Structure et fonctionnement des bases de données ISOE}

\subsubsection{Généralités}

Les quatre bases de données ISOE incluent les données d'exposition professionnelle aux rayonnements ionisants de plus de $90 \%$ des réacteurs nucléaires commerciaux en fonctionnement dans le monde. De ce fait, il s'agit d'un outil unique en son genre d'analyse et de comparaison des pratiques professionnelles et de leurs coûts dosimétriques. À l'heure actuelle, les quatre bases ne sont pas toutes reliées entre elles mais dans un proche avenir, l'ensemble des données devraient coexister sous un environnement Microsoft Access ${ }^{\circledR}$.

Il existe deux niveaux d'accès aux informations des bases de données :

- un niveau complet pour les exploitants participants au programme qui permet d'avoir l'ensemble des données par centrale nucléaire ;

- un niveau restreint pour les autorités de sûreté qui ne donne accès qu'à des moyennes par exploitant.

\subsubsection{Les bases de données ISOE I et ISOE D}

ISOE 1 et ISOE D recueillent les mêmes informations dosimétriques mais la base 1 comporte les données des réacteurs opérationnels alors que la base D (decommissioning) s'intéresse aux réacteurs définitivement arrêtés. Les données enregistrées dans la base pour chaque réacteur participant sont:

- Des données générales concernant le réacteur (type de réacteur, puissance, production électrique annuelle, ...). 
- La dose collective annuelle en fonctionnement normal.

- La dose collective annuelle en arrêt de tranche pour rechargement.

- La dose collective annuelle en arrêt de tranche à la suite d'une anomalie de fonctionnement.

- La dose collective annuelle par type de travail et par type de personnel (mise en place d'échafaudage, isolation thermique, travaux sur la cuve, ...).

\subsubsection{La base de données ISOE 2}

ISOE 2 est une base de données qui comporte des informations plus techniques ayant trait aux moyens pour réduire l'exposition telles que la chimie du réacteur, les procédures de démarrage ou d'arrêt du réacteur, les programmes de réduction du cobalt 60 , l'organisation spécifique du travail pour réduire les expositions...

Actuellement, cette base de données n'a pas encore été intégrée au sein de ISOE 1 mais ceci devrait être réalisé en 2001.

\subsubsection{La base de données ISOE 3}

ISOE 3 est constituée d'un ensemble de textes concis rédigés par les centrales nucléaires. Chaque texte décrit une opération spécifique effectuée au sein de la centrale, son coût dosimétrique et le nom d'une personne à contacter pour des informations complémentaires. L'intérêt de cette base est de permettre un retour d'expérience et donc d'améliorer la réduction et l'optimisation des doses pour l'ensemble des participants au programme.

\subsection{Le réseau de communications du programme ISOE}

Le réseau de communications a pour but de permettre l'échange en temps réel d'informations et d'expériences entre les membres du programme. Le réseau est constitué par l'ensemble des participants et les quatre centres techniques (Fig. 2).

Tout participant peut à travers le réseau poser une question sur un problème technique, une procédure de travail... Cette question est retransmise à l'ensemble des participants à travers son centre technique, qui par ailleurs s'occupe de regrouper les réponses.

\subsection{Les séminaires}

Les deux premiers séminaires organisés par le Centre technique européen en 1998 à Malmö (Suède) et en 2000 à Taragone (Espagne) ont regroupé chacun plus de 150 participants (représentants des exploitants, des autorités réglementaires, 


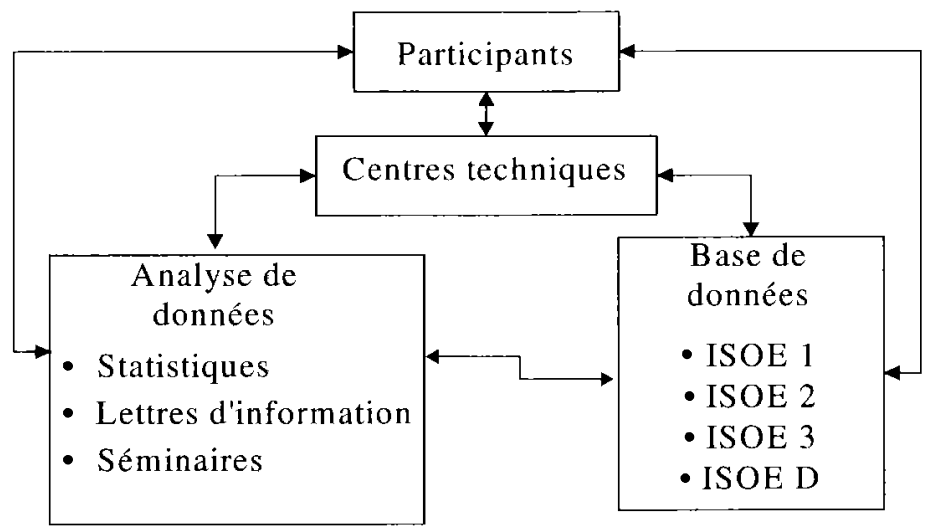

Figure 2 - Le réseau de communication du programme ISOE.

The communication network of the ISOE program.

des entreprises extérieures). De tels séminaires qui permettent à la fois de suivre des exposés et de participer à des ateliers en petits groupes apparaissent comme un moyen privilégié d'échanges entre tous les acteurs de la radioprotection de l'industrie nucléaire.

L'une des principales conclusions et recommandations du dernier séminaire est «qu'il faut envisager de nouvelles techniques de gestion de la radioprotection pour éviter les effets négatifs sur les expositions de la déréglementation du marché de l'électricité et de l'accroissement de la concurrence, tout en maintenant la radioprotection distincte de l'exploitation et de la maintenance des installations ».

L'ensemble des 50 présentations du dernier séminaire seront disponibles d'ici la fin de l'année 2000 sur le site web du CEPN : http://isoe.cepn.asso.fr/. Le prochain séminaire organisé par le centre technique européen aura lieu en 2002 en Slovénie.

\section{ISOE : un outil d'optimisation de l'exposition}

La base de données ISOE 1 permet par l'ensemble des valeurs et informations qu'elle contient d'effectuer des études et comparaisons sur l'exposition professionnelle dans différents pays ou au sein d'un même pays. Afin de pouvoir effectuer des comparaisons informatives pour des réacteurs de conception identique ou différente a été mis en place un système de codification des réacteurs tenant compte à la fois du choix technologique et du nombre de boucles (système des « sister unit group »). 


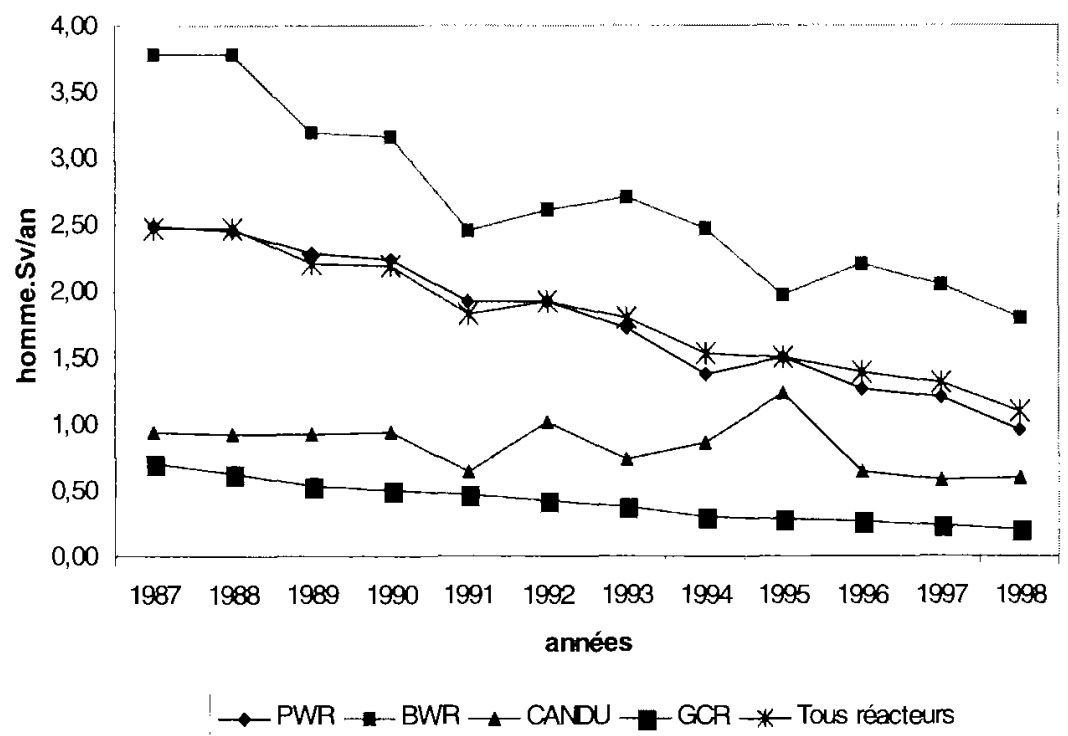

Figure 3 - Dose collective annuelle moyenne par type de réacteur inclus dans la base de données ISOE. Average annual collective dose by reactor type included in the ISOE database.

\subsection{Quelques analyses faites à partir de la base de données}

\subsection{1. Évolution des doses collectives annuelles moyennes par type de réacteur}

ISOE est utilisée pour analyser l'évolution de la dose collective annuelle moyenne par type de réacteur opérationnel inclus dans la base de données (PWR : réacteur à eau sous pression; BWR : réacteur à eau bouillante ; Candu : réacteur modéré à l'eau lourde ; CGR : réacteur graphite gaz). La figure 3 indique clairement que depuis 12 ans pour tous les types de réacteur on assiste à une diminution de l'exposition collective. Cette diminution de l'exposition est bien sûr due à une amélioration de la démarche ALARA mais aussi à une prise de conscience par les opérateurs des différences existant entre eux.

\subsection{2. Évolution de la dose collective annuelle moyenne par pays pour les réacteurs à eau sous pression (PWR)}

Si l'on s'intéresse à un type de technologie de réacteur donné, on peut effectuer des comparaisons beaucoup plus instructives puisqu'elles permettent à un pays de se positionner par rapport aux autres mais aussi de vérifier que sa politique de réduction des doses est en cohérence avec ce qui se fait dans les autres pays. 


\section{homme.Sv/an}

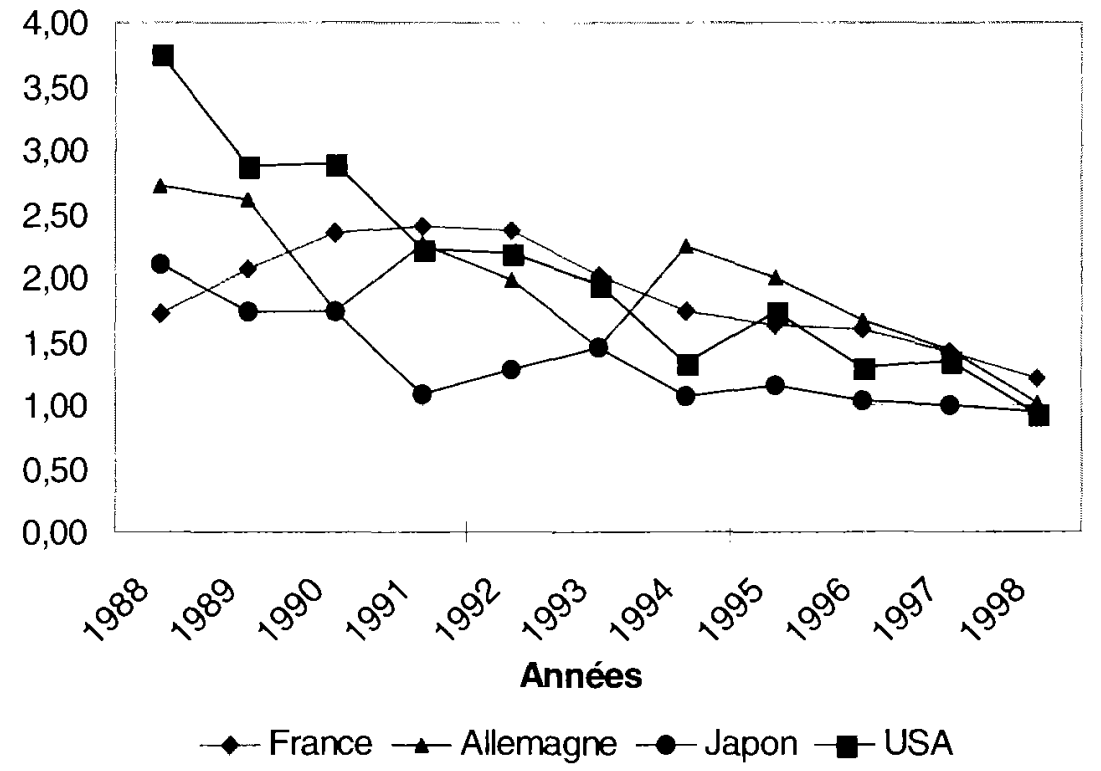

Figure 4-Dose collective annuelle moyenne par pays dans les PWR.

Average annual collective dose by country in PWR.

La figure 4 met en évidence que la dose collective annuelle moyenne par réacteur dans les PWR diminue depuis 10 ans et qu'elle tend à devenir la même pour tous les pays. Ces deux points s'expliquent par une démarche ALARA généralisée, une meilleure organisation du travail, mais aussi par l'allongement des campagnes de combustible (temps entre deux arrêts pour renouvellement du combustible).

\subsubsection{I. Évolution du coût dosimétrique par type de travail : exemple de l'évolution de la dose collective moyenne pour le calorifugeage dans les centrales PWR}

a) Généralités

Les analyses par type de travail sont très intéressantes car elles permettent d'étudier séparément les différents travaux effectués essentiellement durant les arrêts de tranche aux échelons international, national, ou par « sister unit group ». La difficulté majeure dans la réalisation de ces analyses est l'extraction des données car même s'il existe une sorte de charte, pour les centrales, sur la manière de remplir les questionnaires sous Access ${ }^{\circledR}$, il n'en reste pas moins qu'il est difficile de juger de la validité des doses données par type de travail. Par exemple, 


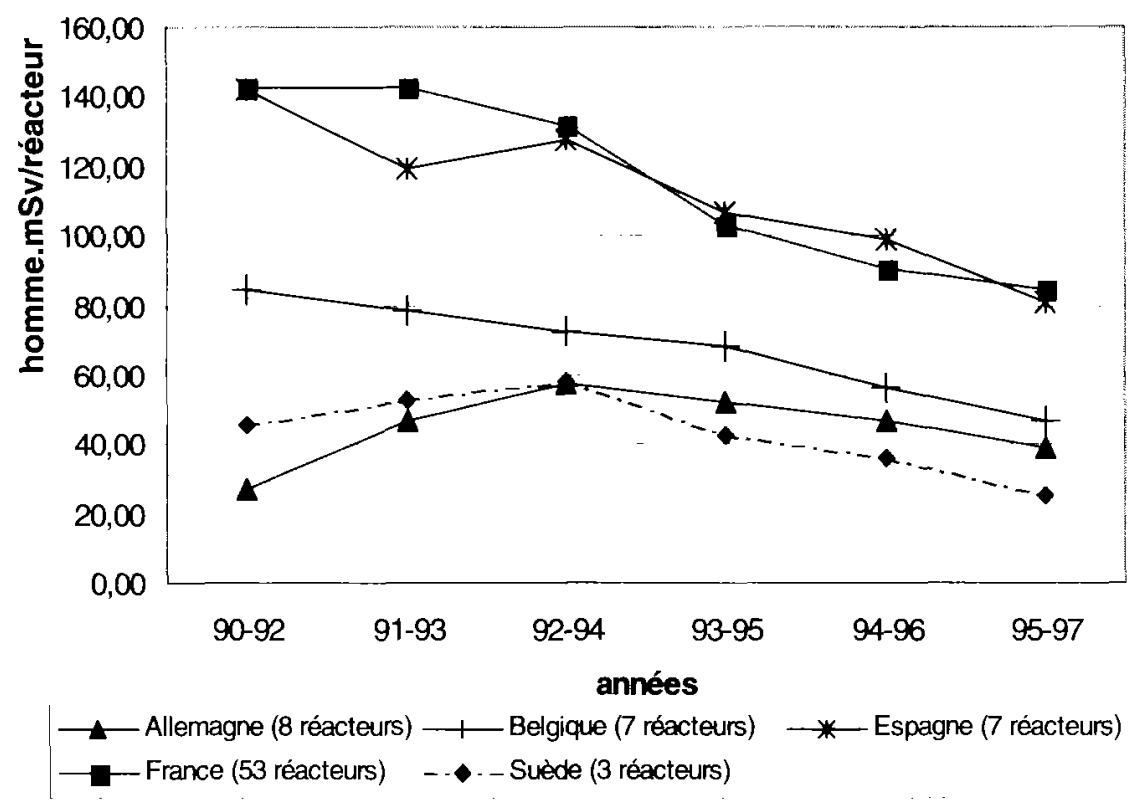

Figure 5 - Dose collective moyenne par pays (nombre de réacteurs concernés) pour le calorifugeage des PWR (moyenne glissante sur trois ans).

Average collective dose (three years rolling average) for PWR insulation by country (number of unit concerned).

dans les doses par type de travail «calorifugeage », une partie de la dose peut être due à la mise en place d'échafaudage alors que ce type de travail existe dans une autre rubrique. En somme, si toutes les centrales ne donnent pas de la même façon les doses par type de travail, il existe alors un biais de recueil des données qui pose un problème pour l'interprétation.

b) Évolution de la dose collective moyenne pour le calorifugeage dans les centrales PWR

Cette étude a été réalisée sur l'ensemble des réacteurs européens à eau sous pression pour lesquels on disposait de suffisamment de données pour en faire une exploitation statistique. Le choix de l'utilisation de moyennes glissantes sur trois ans s'explique par l'allongement de l'intervalle de temps entre deux rechargements de réacteur (dix-huit mois voire deux ans). La figure 5 permet d'observer qu'il existe une tendance générale à la baisse de la dose collective due au calorifugeage dans tous les pays. Il est intéressant de noter que cette baisse est 


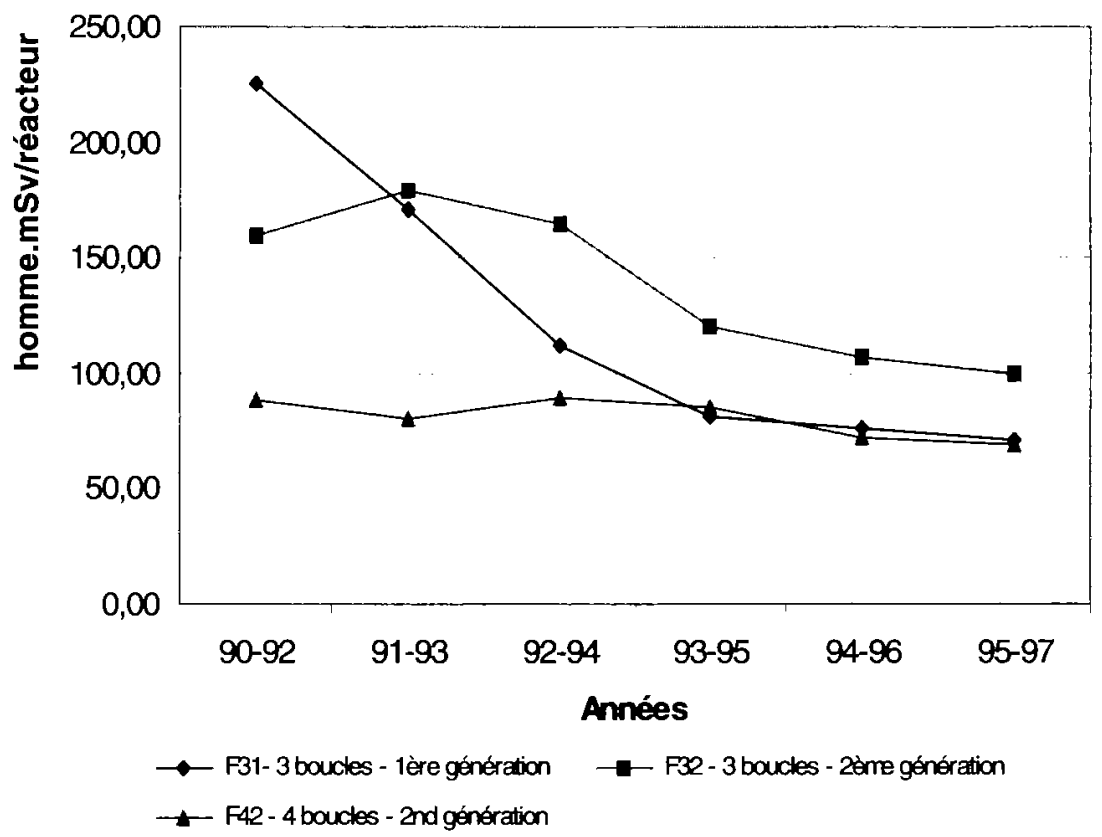

Figure 6-Dose collective moyenne pour le calorifugeage des centrales Framatome (moyenne glissante sur trois ans).

Average collective dose for insulation work in Framatome plants (three years rolling average).

en fait tout à fait corrélée à la diminution de la dose collective annuelle au sein de chaque pays pour la même période.

Si ces données générales sont intéressantes, elles ne permettent pas en revanche de mettre en évidence l'impact d'une part des pratiques et d'autre part de la technologie utilisée. Le facteur technologique est très bien identifié par l'utilisation des «sister unit group» comme on peut le voir sur les figures 6 et 7 qui présentent la dose collective moyenne due au calorifugeage dans les centrales nucléaires de technologie Framatome et dans celles de technologie Siemens.

Par ailleurs, pour les premières, on observe qu'il existe un facteur « pratique » très important puisque la dose collective moyenne au sein du «sister unit group » F31 (F31 signifie réacteur à 3 boucles de première génération) a été divisée par un facteur trois en l'espace de 10 ans (Fig. 6). Les changements de pratiques ont parfaitement pu être identifiés grâce à un questionnaire spécifique, distribué dans les centrales concernées, sur les méthodes de calorifugeage et leurs évolutions récentes. 


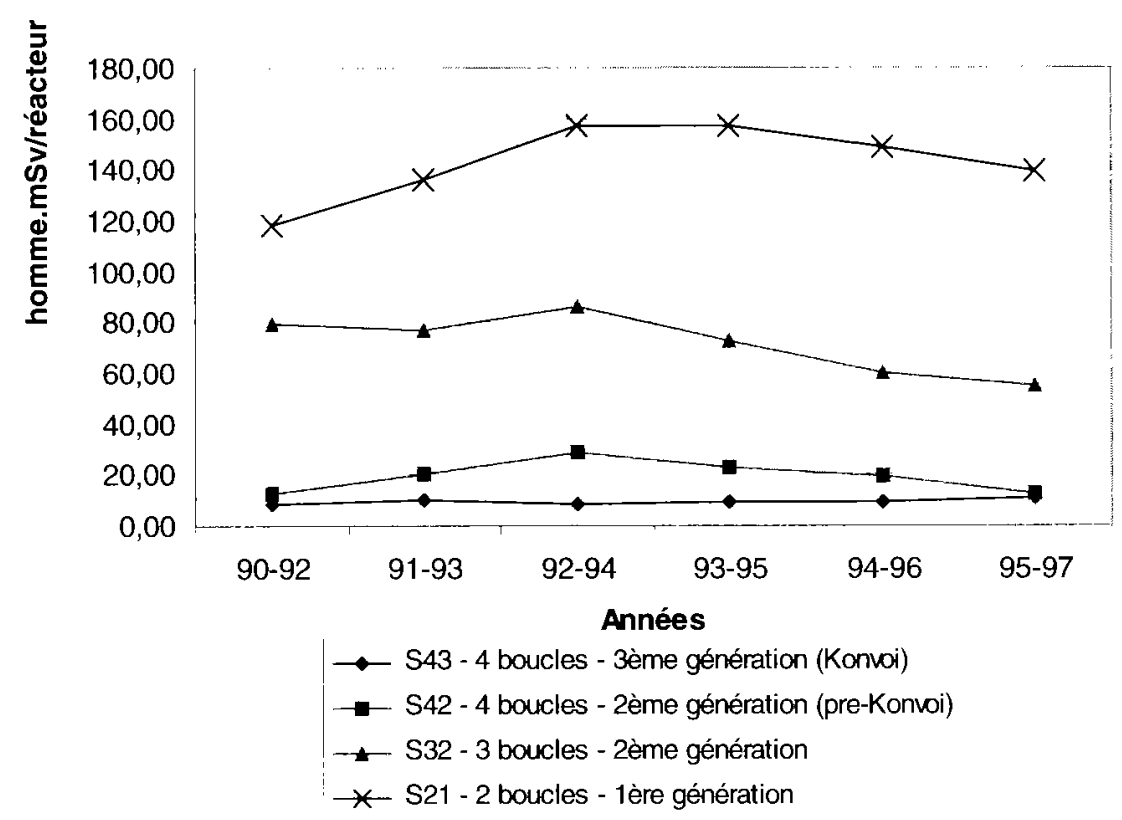

Figure 7 - Dose collective moyenne pour le calorifugeage des centrales de conception Siemens (moyenne glissante sur trois ans).

Average collective dose for insulation work in Siemens plants (three years rolling average).

\section{Mise en perspective}

Chaque année la base de données ISOE s’enrichit de la dosimétrie collective de plus de 150000 travailleurs ainsi que d'informations spécifiques sur les méthodes utilisées par chaque pays pour réduire les doses. Le volume de données et le fait que celles-ci soient recueillies partout de la même façon aboutissent à un outil exemplaire en matière de suivi de l'exposition professionnelle.

Les améliorations récentes apportées au programme permettent d'obtenir plus facilement des données exploitables et intéressantes pour ce qui est des données globales. En revanche, les études par type de travail demandent toujours un effort important, d'une part pour l'extraction des données, et, d'autre part, pour leur validation. En effet, il existe des différences individuelles dans la manière de répondre au questionnaire qui aboutissent parfois à une information, soit tronquée, soit dispersée dans la base de données. Ces défauts de jeunesse ne doivent pas faire perdre de vue qu'ISOE est avant tout un formidable outil pour l'amélioration de la protection radiologique des travailleurs et que de tels outils pourraient être développés dans d'autres grands domaines industriels. 


\section{Conclusion}

Le système de retour d'expérience ISOE, créé en 1992, représente une avancée très importante dans le domaine de la radioprotection opérationnelle, tant par le biais de la base de données que par celui du réseau et des séminaires. En effet, si plusieurs exploitants possédaient déjà, il y a quelques années, des outils de comparaison des pratiques au sein de leurs centrales, en revanche rien n'existait sur le plan international. Grâce à la base de données et au système ISOE, on dispose à l'heure actuelle d'un outil unique pour l'étude et la comparaison des pratiques en radioprotection pour plus de $90 \%$ des réacteurs nucléaires commerciaux en fonctionnement. Ces comparaisons peuvent se faire sur un plan purement factuel ou au contraire de manière analytique de façon à profiter au mieux de l'expérience commune et donc se rapprocher le plus possible du «achievable » du principe ALARA.

\section{RÉFÉRENCES}

ISOE (1993) Nuclear power plant occupational exposures in OECD countries: 1969-1991, OECD.

ISOE (1994) Nuclear power plant occupational exposures in OECD countries: 1969-1992, OECD.

ISOE (1995) Third annual report: Occupational exposures at nuclear power plants: 1969-1993, OECD.

ISOE (1996) Fourth annual report: Occupational exposures at nuclear power plants: 1969-1994, OECD.

ISOE (1997) Fifth annual report: Occupational exposures at nuclear power plants: 1969-1995, OECD.

ISOE (1998) Sixth annual report: Occupational exposures at nuclear power plants: 1969-1996, OECD.

ISOE (1999) Scventh annual report of the ISOE programme: Occupational exposures at nuclear power plants: 1997, OECD.

ISOE (1999) Eighth annual report of the ISOE programme: Occupational exposures at nuclear power plants: 1998, OECD.

Mundigl S., Gustafsson M. (2000) The information system on occupational exposure (ISOE): present and future activities in: 2nd EC/ISOE workshop on occupational exposure management at NNP's Tarragona, Spain, 5-7 April 2000.

Mundigl S., Lazo T. (1999) ISOE studies show local effect on dose management, Nuclear Engineering International 44-544, 32-33.

Work Management in the Nuclear Power Industry (1997) OECD. 